\title{
Melter, Claus
}

\section{Kritische Soziale Arbeit in Diskriminierungs- und Herrschaftsverhältnissen - eine Skizze}

Spetsmann-Kunkel, Martin [Hrsg.]; Frieters-Reermann, Norbert [Hrsg.]: Soziale Arbeit in der Migrationsgesellschaft. Opladen ; Berlin ; Toronto : Verlag Barbara Budrich 2013, S. 93-111. - (Schriften der Katholischen Hochschule Nordrhein-Westfalen; 17)

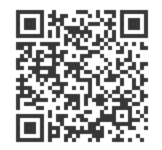

Quellenangabe/ Reference:

Melter, Claus: Kritische Soziale Arbeit in Diskriminierungs- und Herrschaftsverhältnissen - eine Skizze - In: Spetsmann-Kunkel, Martin [Hrsg.]; Frieters-Reermann, Norbert [Hrsg.]: Soziale Arbeit in der Migrationsgesellschaft. Opladen ; Berlin ; Toronto : Verlag Barbara Budrich 2013, S. 93-111 - URN: urn:nbn:de:0111-pedocs-127878 - DOI: 10.25656/01:12787

https://nbn-resolving.org/urn:nbn:de:0111-pedocs-127878

https://doi.org/10.25656/01:12787

in Kooperation mit / in cooperation with:

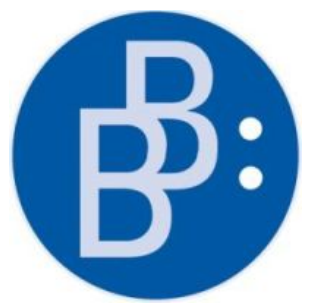

https://www.budrich.de

\section{Nutzungsbedingungen}

Dieses Dokument steht unter folgender Creative Commons-Lizenz: $\mathrm{http}: / /$ creativecommons.org/licenses/by-nc-nd/3.0/de/deed - Sie dürfen das Werk bzw. den Inhalt unter folgenden Bedingungen vervielfältigen, verbreiten und öffentlich zugänglich machen: Sie müssen den Namen des Autors/Rechteinhabers in der von inm festgelegten Weise nennen. Dieses Werk bzw. dieser Inhalt darf nicht für kommerzielle Zwecke verwendet werden und es darf nicht bearbeitet, abgewandelt oder in anderer Weise verändert werden.

Mit der Verwendung dieses Dokuments erkennen Sie die Nutzungsbedingungen an.

\section{Terms of use}

This document is published under following Creative Commons-License: http://creativecommons.org/licenses/by-nc-nd/3.0/de/deed.en - You may copy, distribute and transmit, adapt or exhibit the work in the public as long as you attribute the work in the manner specified by the author or licensor. You are not allowed to make commercial use of the work or its contents. You are not allowed to alter, transform, or change this work in any other way.

By using this particular document, you accept the above-stated conditions of use.

\section{Kontakt / Contact:}

peDOcs

DIPF | Leibniz-Institut für Bildungsforschung und Bildungsinformation

Informationszentrum (IZ) Bildung

E-Mail: pedocs@dipf.de

Internet: www.pedocs.de

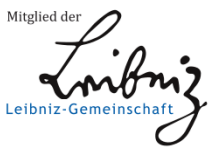


Martin Spetsmann-Kunkel

Norbert Frieters-Reermann (Hrsg.)

\section{Soziale Arbeit in der Migrationsgesellschaft}

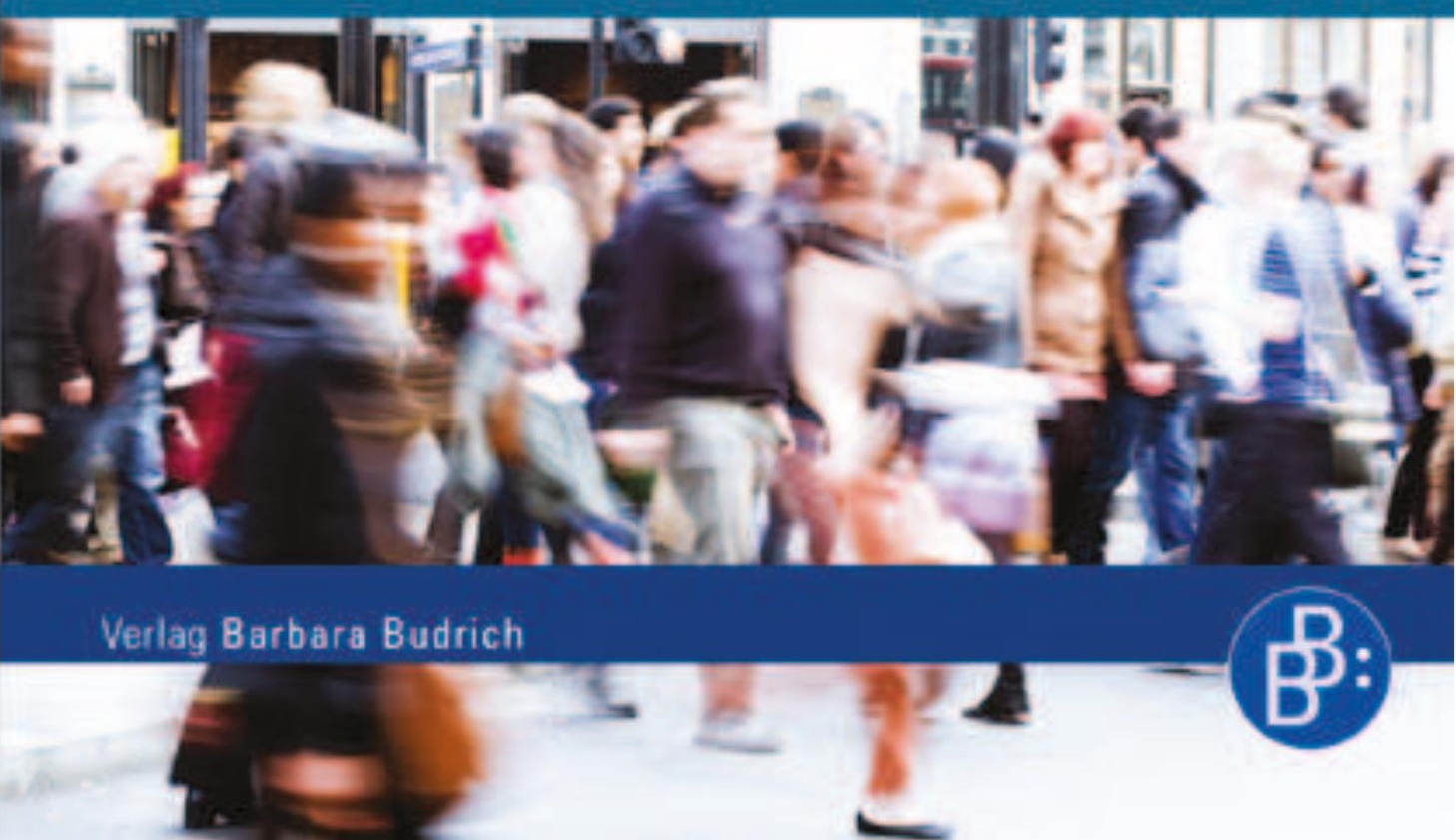


Schriften der Katholischen Hochschule

Nordrhein-Westfalen

Band 17

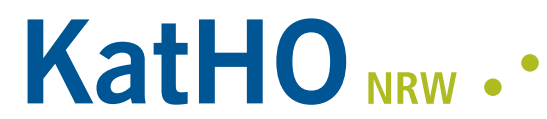

Aachen | Köln | Münster | Paderborn

Katholische Hochschule Nordrhein-Westfalen Catholic University of Applied Sciences 
Martin Spetsmann-Kunkel

Norbert Frieters-Reermann (Hrsg.)

\section{Soziale Arbeit in der} Migrationsgesellschaft

Verlag Barbara Budrich

Opladen, Berlin \& Toronto 2013 
Prof. Dr. Martin Spetsmann-Kunkel

Prof. Dr. Norbert Frieters-Reermann

Katholische Hochschule NRW / Aachen

Robert-Schuman-Straße 25

52066 Aachen

Tel.: (+49) (0)241-6000318

Bibliografische Information der Deutschen Nationalbibliothek

Die Deutsche Nationalbibliothek verzeichnet diese Publikation in der Deutschen

Nationalbibliografie; detaillierte bibliografische Daten sind im Internet über

http://dnb.d-nb.de abrufbar.

(C) 2013 Dieses Werk ist im Verlag Barbara Budrich erschienen und steht unter folgender Creative Commons Lizenz: http://creativecommons.org/licenses/by-nc$\mathrm{nd} / 3.0 / \mathrm{de} /$

Verbreitung, Speicherung und Vervielfältigung erlaubt, kommerzielle Nutzung und Veränderung nur mit Genehmigung des Verlags Barbara Budrich

Dieses Buch steht im OpenAccess Bereich der Verlagsseite zum kostenlosen Download bereit (https://doi.org/10.3224/93809468)

Eine kostenpflichtige Druckversion kann über den Verlag bezogen werden. Die Seitenzahlen in der Druck- und Onlineversion sind identisch.

\section{ISBN 978-3-938094-68-6 (Paperback) \\ eISBN 978-3-8474-0337-1 (eBook) \\ DOI $\quad 10.3224 / 93809468$}

Umschlaggestaltung: disegno visuelle kommunikation, Wuppertal - www.disenjo.de Typografisches Lektorat: Petra Reiners, Bonn - www.buchfinken.com Druck: paper \& tinta, Warschau

Printed in Germany 


\section{Inhaltsverzeichnis}

1. Soziale Arbeit in der Migrationsgesellschaft einleitende Gedanken

Martin Spetsmann-Kunkel

2. Anfragen an die Praxis der Sozialen Arbeit in einer von Mobilität und Vielfalt geprägten Postmoderne - Soziale Arbeit in der Migrationsgesellschaft

Wolf-D. Bukow

3. Interkulturelle Soziale Arbeit: Integration, Anerkennung und Partizipation als Leitideen einer differenzsensiblen Sozialen Arbeit in der Migrationsgesellschaft

Josef Freise

4. Interkulturelles Lernen und Forschen in transkulturellen Dialoggruppen

Cornelia Muth

5. Demokratiepädagogik und Diversity Education pädagogische Konzepte und ihre Bedeutung für die Soziale Arbeit

Schahrzad Farrokhzad 
6. Kritische Soziale Arbeit in Diskriminierungs- und Herrschaftsverhältnissen - eine Skizze

Claus Melter

7. Soziale Arbeit in mobiler Gesellschaft Institutionelle Bedingungen, professionelle Expertise und das eigene Verschränkt-Sein in Alltagsrassismus und kulturalisierenden Deutungsprozessen

Claudia Roller

8. Interkulturelle Öffnung als Team - von Stolpersteinen und Meilensteinen

Norbert Teutenberg

9. Die Migrationssozialarbeit der Caritas

Thomas Kley

10. Soziale Arbeit und Migration - Auslassungen, Anregungen und Ausblicke

Norbert Frieters-Reermann 


\title{
6. Kritische Soziale Arbeit in Diskriminierungs- und Herrschaftsverhältnissen - eine Skizze
}

\author{
Claus Melter
}

Im Folgenden sollen Diskriminierungs- sowie rassismus- und herrschaftskritische Grundaussagen im Sinne vorgeschlagener Begriffsverständnisse und Interpretationen dargelegt und dann Ideen für eine diskriminierungs- sowie herrschaftskritische Pädagogik im Kontext von Migrationsgesellschaft, Diskriminierung und Rassismus konzipiert werden.

\section{Diskriminierungs- sowie rassismus- und herrschaftskritische Grundaussagen und Analysen}

Stereotype und Vorurteile können als verallgemeinernde Gruppenkonstruktionen und Vorurteile als verallgemeinernde Gruppenkonstruktionen zusätzlich mit Negativurteilen verstanden werden (vgl. Zick 1997), die möglicherweise ausschließlich im Kopf von Personen stattfinden. Diskriminierungen beinhalten darüber hinaus oder auch unabhängig davon beobachtbare Handlungen oder die Effekte von Handlungen, Regelungen und Ressourcenverhältnissen, die bestimmte Gruppen oder Personen in Bezugnahme auf ihre Gruppenangehörigkeit benachteiligen. So kann in Bezug auf die Stephen Lawrence Inquiry und das Allgemeine Gleichbehandlungsgesetz gefragt werden: Wer hat Zugang zur Organisation als Mitarbeitende, Adressat/innen oder auch Träger/innen und Geldgebende oder Gesetz- und Regelvorgebende? Und erhalten Personen und Gruppen den gleichen professionellen Service wie ihn Angehörige anderer Gruppen erhalten oder entsprechend der allgemeinen Regelungen erwarten können und können sie in gleicher Weise mitwirken?

In Anlehnung und Erweiterung des analytischen Grundmodells von Albert Scherr (2010) schlage ich folgendes analytisches Modell vor (Abb. 1): 
Abb. 1: Analytisches Grundmodell

\section{Analytisches Grundmodell}

Ökonomisch-materielle

Kapital-und Produktionsverhältnisse

und Klassen der unterschiedlich

Besitzenden

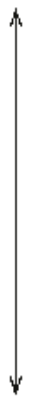
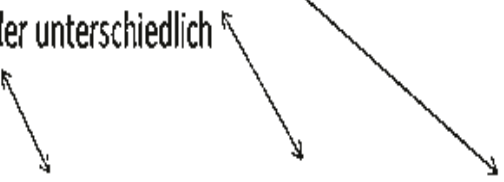

$\left\{\begin{array}{l}\text { materiellen } \\ \text { Ressourcen }\end{array}\right.$

Diskurseüber -

1) Institutionelle

Ungleichere

Gerechtigkeits- $\longleftrightarrow$ Praxen $\longleftrightarrow$ Zugangzu

institutionell

Macht

\section{vorstellungen 2| Wirtschaft}

Diskriminierende Unter-

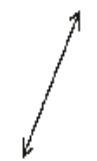

scheidungen auf der

Grundlagevon Gruppenkonstraktionen

(informell und formally und Zugehörigkeits.

zuschreibungen
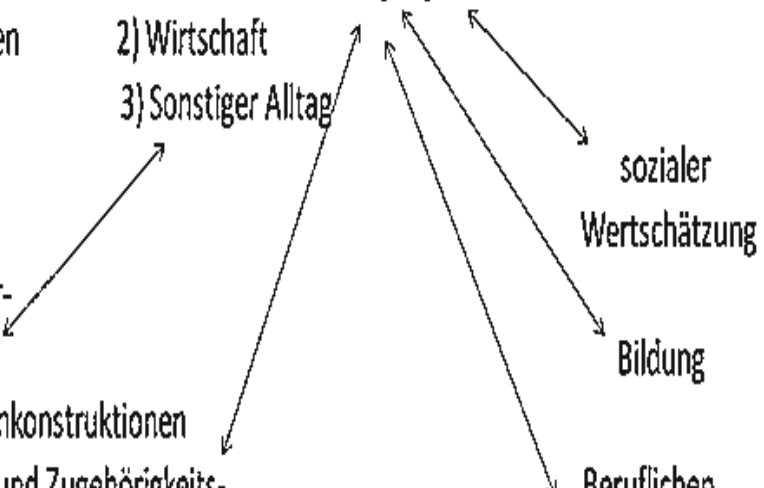

sozialer

Wertschätzung

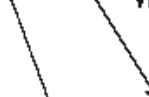

Bildung

Beruflichen

Karrieren

Erwerbsarbeit

(Melter 2012: Modell in Anlehnung an Scherr 2010)

Um das Modell skizzenhaft zu erläutern: Als relevanter Einfluss in Gesellschaften sind die ökonomisch-materiellen (also auch finanziellen) Kapi- 
tal- und Produktionsverhältnisse und die daraus resultierenden Klassen der unterschiedlich Besitzenden zu sehen. Diese bestehen jedoch nicht an sich oder unabhängig von sozialen Gruppenkonstruktionen (Geschlecht, Ethnie, Staatsbürger/innenschaft, Rassialisierungen, Behinderung, sexueller Orientierung, Alter usw.), sondern sind mit ihnen verwoben. So kann von einem ethnisierten und vergeschlechtlichten Arbeitsmarkt gesprochen werden, auf dem z.B. als mehrheitsangehörig angesehene Personen in vielen Berufen privilegiert werden. Ebenso gibt es viele Belege für die Diskriminierung von Schüler/innen aus wirtschaftlich ärmeren Familien und mit zugeschriebener Migrationsgeschichte (vgl. Dirim/Mecheril 2010, Melter/Karayaz 2013). Mehrgliedrige Schulsysteme mit dominant schulklassen-bezogener und nicht individueller oder kleingruppen-bezogener Didaktik und gemeinsamem Lernen nur bis zur vierten oder sechsten Klassen (Herzog-Punzenberger/Schnell 2011) bewirken in diesem Kontext sowohl die Diskriminierung von Menschen mit zugeschriebener Migrationsgeschichte und aus wirtschaftlich schwächeren Familien als auch von Schüler/innen mit zugeschriebener Behinderung (Eckhart u.a. 2011). Schulerfolge beeinflussen die Chancen auf dem Arbeitsmarkt, allerdings gibt es ethnisierende/rassialisierende sowie nationenbezogene Diskriminierung trotz guter Schulnoten (vgl. Gächter 2010). Bildungserfolg schützt nicht vor Diskriminierung auf dem Arbeitsmarkt.

Die Ressourcenverhältnisse (bezogen auf ökonomisch-materielles, soziales, kulturelles und symbolisches Kapital; vgl. Bourdieu 1992) sind von Ungleichheit gekennzeichnet. So besteht in Deutschland die zunehmende Entwicklung, dass die finanziell reichen Haushalte immer reicher und die ärmeren Haushalte und darin lebenden Personen zunehmend ärmer werden. So besitzen die unteren fünfzig Prozent der Haushalte nur ein Prozent des Gesamtfinanzvermögens und die oberen 10 Prozent 53 Prozent (vgl. Frankfurter Rundschau 19.09.2012, S. 2). Begleitet wird dies von Diskursen einer Kulturalisierung der Armut (vgl. Kessl u.a. 2007), in dem den weniger Besitzenden und weniger Erfolgreichen und ihren Lebenspraxen und Einstellungen (ihrer „Kultur") alleine die Verantwortung für ihre Lebenslage zugeschrieben wird. Klassen-, Macht- und Diskriminierungsverhältnisse werden in Diskursen über Gerechtigkeitsvorstellungen tendenziell unter alleiniger Bezugnahme auf Leistungsgerechtigkeit im meritokratischen Sinne und unter Nicht-Beachtung der Themen Ressourcenverteilungs-, Zugangsund Befähigungs-/Förderungsgerechtigkeit individualisiert, kulturalisiert, ethnisiert sowie verschleiert oder geleugnet.

Dem gegenüber gibt es Forderungen nach mehr Verteilungs-, Zugangsund Befähigungsgerechtigkeit. Letztere wird entsprechend der Ottawa-Charta definiert als Möglichkeit, ,selbst Entscheidungen zu fällen und eine Kontrolle über die eigenen Lebensumstände auszuüben sowie darum, das die 
Gesellschaft, in der man lebt, die Bedingungen herstellt, die allen Bürgern Gesundheit ermöglichen" (vgl. Bundesministerium für Familie, Senioren, Frauen und Jugend 2009, S. 38). In einer Wirkungs-, Gesellschafts- und Institutionen bezogenen Diskriminierungsdefinition werden Verhältnisse und Handlungen sowie die Prozesse in Institutionen, die gemeinsam unterschiedliche Ressourcenzugänge bewirken, als diskriminierend benannt. Faktisch können in Deutschland und vielen anderen Ländern unterschiedliche $\mathrm{Zu}$ gänge zu materiellen und finanziellen Ressourcen, zu sozialer Wertschätzung, zu Bildungs- und Arbeitsmöglichkeiten sowie zu institutionell politischer Macht konstatiert werden. All diese Faktoren und Aushandlungsprozesse bewirken einen ungleichen Zugang zu materiellen und finanziellen Ressourcen, zu institutionell politischer Macht, sozialer Wertschätzung, beruflichen Karrieren und Arbeitsmöglichkeiten.

Wir leben und arbeiten in Diskriminierungsverhältnissen, die durch unterschiedliche Gerechtigkeitsforderungen wenig effektiv kritisiert werden.

\section{Funktionen und Positionierungen Sozialer Arbeit in Diskriminierungsverhältnissen - die Aspekte Religion und Kirche als Wohlfahrtskonzern}

Soziale Arbeit ist organisationsbezogen und ökonomisch einerseits gekennzeichnet durch das Quasi-Monopol des Staates als Finanzregulierer, Auftraggeber und gesetzlicher Rahmengeber. Andererseits dominieren die Wohlfahrtskonzerne Arbeiterwohlfahrt, Deutscher Paritätischer Wohlfahrtsverband sowie Caritas und Diakonie diese Arbeitsfelder (vgl. Wulf-Schnabel 2010). Die kirchlichen Wohlfahrtskonzerne operieren ebenso wie die kirchlichen Hochschulen mit Praxen der religiösen Diskriminierung, indem fast ausschließlich Angehörige christlicher Konfessionen angestellt werden. Hierdurch sowie durch staatsbürgerliche und ethnisierende/rassialisierende Mechanismen werden ganze Arbeitsfelder der Sozialen Arbeit vorwiegend von Mehrheitsangehörigen und christlichen Mitarbeiter/innen dominiert. Unabhängig von der Professionalität und dem guten Willen der Mitarbeiter/innen werden Vorstellungen von interreligiösem oder interkulturellem Dialog und von Zugangs-, Verteilungs- und Befähigungsgerechtigkeit durch diese Diskriminierungspraxen erschwert bis verunmöglicht bzw. werden von nichtchristlichen Personen als machtvoll oder diskriminierend erlebt. Gleiches gilt für interreligiös-staatliche Veranstaltungen wie die Islamkonferenzen (vgl. Badawia 2009). Ermöglicht wird die religiöse Diskriminierung, die mit ethnisierenden und staatsbürgerschaftsbezogenen Diskriminierungspraxen (z.B. 
durch fehlende oder erschwerte Anerkennung von Ausbildungen und eingeschränkten Aufenthaltsstatus) verwoben ist, durch Lücken im Allgemeinen Gleichbehandlungsgesetz, in dem der Staat - entgegen den Forderungen in der Allgemeinen Erklärung der Menschenrechte - Nationen-bezogene Diskriminierung nicht als Kriterium gelten lässt (vgl. Frings 2008). Zudem hat der Gesetzgeber dem Druck der christlichen Kirchen nachgegeben, indem er religiöse Diskriminierung nicht als uneingeschränktes Kriterium im Allgemeinen Gleichbehandlungsgesetz aufgenommen hat. Die ersten beiden Artikel der Allgemeinen Erklärung der Menschenrechte lauten demgegenüber:

„Artikel 1: Alle Menschen sind frei und gleich an Würde und Rechten geboren. Sie sind mit Vernunft und Gewissen begabt und sollen einander im Geiste der Brüderlichkeit begegnen. Artikel 2: Jeder hat Anspruch auf alle in dieser Erklärung verkündeten Rechte und Freiheiten, ohne irgendeinen Unterschied, etwa nach Rasse ${ }^{4 P}$, Hautfarbe, Geschlecht, Sprache, Religion, politischer oder sonstiger Anschauung, nationaler oder sozialer Herkunft, Vermögen, Geburt oder sonstigem Stand" (Generalversammlung der Vereinten Nationen, 10.12.1948).

In einem von Dorothee Frings im Auftrag der Antidiskriminierungsstelle des Bundes erstellten Gutachten heißt es:

„Religionsgemeinschaften und religiös ausgerichtete Wohlfahrtsverbände dürfen das Bekenntnis zu ihrer Religion für eine Beschäftigung voraussetzen, wenn die Tätigkeit oder die Umstände der Ausübung in einem Zusammenhang mit dem religiösen Auftrag der Organisation stehen. Dieser Auftrag wird von den Religionsgemeinschaften autonom bestimmt und kann nicht auf seine Angemessenheit hin überprüft werden. Steht die Tätigkeit nicht in einer Beziehung zu dem religiösen Auftrag, sind Ungleichbehandlungen wegen der Religion untersagt. § 9 AGG muss insoweit an die Vorgaben der europäischen Rechtssetzung angepasst werden“" (Frings/Antidiskriminierungsstelle des Bundes 2010, S. 2).

Auch Nickel verweist darauf, dass die Rechtslage und Rechtsprechung in Deutschland gegen europäisches Recht und europäische Rechtsprechung verstößt (vgl. Nickel 2010). Es liegt also an Gesetzgeber, Kirchen und konkret ihren Mitarbeiter/innen vor Ort auch in den Einrichtungen Sozialer Arbeit die bestehende diskriminierende Praxis so zu verändern, dass bestehende gelingende Arbeit - die es zweifelsohne gibt - im Sinne von mehr Gerechtigkeitsorientierung verändert wird.

41 Es verwundert, dass die Vereinten Nationen einerseits die Existenz von „Rassen“ als wissenschaftlich widerlegt und politisch als gefährlich darlegen und andererseits den Begriff und das Konzept ohne Kommentierung in anderen Dokumenten verwenden. Ähnliches lässt sich über das Grundgesetz sagen. 


\section{Ökonomische Dominanz als Handlungsorientierung erschwert professionelle Arbeit mit den Adressat/innen}

Organisationen und Mitarbeiter/innen haben - so zeigen Forschungen zu institutioneller Diskriminierung in den Bereichen Bildung und Soziale Arbeit - als oberste Ziel, den betrieblichen Anforderungen zu entsprechen und den Bestand der eigenen Organisation fortzuführen. Diese ,wettbewerbliche Subjektivierung“ (Wulf-Schnabel 2011) dominiert die ,gefangene Subjektivierung" (gemeint ist die Arbeit mit den Adressat/innen im Sinne einer bedürfnisorientierten Förderung und Unterstützung ihrer Interessen) und führt oftmals zu einer tendenzielle Aufgabe des politischen Mandats bzw. die Delegierung dieser Aufgabe an Funktionär/innen (vgl. Wulf-Schnabel 2011).

Die großen Wohlfahrtskonzerne ${ }^{42}$ sind auch nach dem „sozialpädagogischen Jahrhundert“ (Thomas Rauschenbach) weiter im Sinne von Angeboten und Personen am Wachsen und so gehören sie zum Teil zu den bundesweit größten Arbeitgebern im gesamten Wirtschaftsbereich. Gleichzeitig sind im Rahmen neoliberaler Umstrukturierungen und des Abbaus des Sozialstaates einzelne Einrichtungen und Angebote und somit die in diesen Angeboten Beschäftigten mit verstärkten Leistungsanforderungen sowie Kürzungs- und Streichungsandrohungen einiger Angebote konfrontiert. Jan Wulf-Schnabel untersucht in seiner lesenswerten Studie zu „Reorganisation und Subjektivierungen von Sozialer Arbeit“ (Wulf-Schnabel 2011) die Umstrukturierung und Arbeitspraxen eines großen Leistungsträgers im Sozialbereich - die AWO in Schleswig Holstein - wie dies von den Arbeitenden wahrgenommen und umgesetzt wird. Eine Besonderheit dieser Arbeit ist, dass systematisch Genderaspekte in der Analyse von Arbeitsplatzverschiebungen und Subjektivierungsprozessen im Rahmen der Reorganisation berücksichtigt werden. Eine zentrale These der Arbeit von Wulf-Schnabel ist die antagonistische Gegenüberstellung von ,gefangener Subjektivierung“", die als originäres Charakteristikum Sozialer Arbeit durch den Koproduktionscharakter Sozialer Arbeit und die sowohl fehlende oder schwer zu messende Produkterstellung und den Verkauf an Zweite oder Dritte angesehen wird, zu wettbewerblicher Subjektivierung, die auf Marktund Konkurrenzverhältnisse orientiert.

„Aufgrund eines sozialpolitischen Paradigmenwechsels bestimmen Kostensenkungen und Effizienzmaßstäbe die Arbeitsbeziehung und die Arbeit wird stärker vom Ergebnis gesteuert. In der Folge werden die

42 Teile des folgenden Absatzes sind in ähnlicher Form bereits publiziert worden (vgl. Melter 2011a). 
Arbeitenden mit gestiegenen Anforderungen an ihre Produktivität konfrontiert und so auf sich selbst und die Wirtschaftlichkeit ihrer Leistungen verwiesen. Die Arbeitenden müssen nunmehr wettbewerbliche Subjektivierungsleistungen erbringen, die, so die zweite These, in einem grundlegenden Widerspruch zur gefangenen Subjektivierung stehen" (WulfSchnabel 2011, S. 17) und letztere zunehmend dominieren.

Wie kann eine fachlichen Standards entsprechende, adressat/ innenorientierte sowie diskriminierungskritische und am politischen Mandat gegen Benachteiligung interessierte Soziale Arbeit mit diesen Entwicklungen umgehen?

\section{Diskriminierung und Herrschaft}

Ein wichtiger Schritt wird sicherlich eine theoretische und mit den Adressat/innen zu erfolgende Auseinandersetzung mit Diskriminierungs-Praxen sein. Diskriminierungskritische Soziale Arbeit, so schlage ich vor, versteht unter Diskriminierung sowohl absichtsvoll abwertende, benachteiligende oder ausgrenzende Handlungen als auch Handlungen und Verhältnisse, die in ihren Effekten bestimmte Gruppe systematisch abwerten, benachteiligen oder ausgrenzen (ohne dass dies jeweils absichtsvoll so beabsichtigt wurde) (vgl. Gomolla 2010). Unterschieden werden auf der einen Seite benachteiligende Handlungen, die Menschen erlauben, auf einem Territorium $\mathrm{zu}$ sein und $\mathrm{zu}$ agieren, allerdings nicht mit gleichen Rechten und Ressourcenzugängen und Handlungsmöglichkeiten - dies nenne ich unterwerfende Eingliederung. Auf der anderen Seite gibt es ausgrenzende Handlungen, die den physischen Ausschluss von Menschen von einem territorialen oder sozialen Raum beabsichtigen oder realisieren - z.B. durch Ausweisungen, Abschiebungen oder Nicht-Einlasspraxen an Grenzen oder in Diskotheken oder Vereinen.

Diskriminierungen finden auf struktureller (gesellschaftliche Bildungsund Arbeitsmarkt, Wohn- und Einkommensverhältnisse), institutioneller (Kindergarten, Schule, Hochschule, Polizei, Arbeits-, Sozial- und Ausländerbehörden, Justiz, Gesundheitsbehörden) diskursiver (was im öffentlichen und privaten Raum zu einem Thema gesprochen, geschrieben, sowie in Gesetzen und in Bauwerken realisiert wird) und interaktiver (Handlungen zwischen Menschen) Ebene statt (vgl. Melter 2006). Wenn Diskriminierungen in einer Gesellschaft systematisch und über einen längeren Zeitraum von einer Gruppe, die ihre Interessen machtvoll durchsetzen kann, praktiziert werden, kann von systematisch ungleichen Machtverhältnissen gesprochen werden, also von gesellschaftlichen Herrschaftsverhältnissen. 
Als Herrschaftsverhältnisse, die in der Regel mit zwei- oder mehrteiligen, sich ausschließenden (binären) Gruppenkonstruktionen wie Männer-Frauen, „Inländer/innen“-,,Ausländer/innen“, „Nicht-Behinderte“„Behinderte“, ... operieren, können Sexismus, Rassismus, Behindertenfeindlichkeit und Klassenverhältnisse genannt werden, wobei letztere auf den ungleichen Besitz- und Ressourcenzugangsverhältnissen zu materiellen und ökonomischen Mitteln bestehen. Diese Herrschaftsverhältnisse sind miteinander verwoben (Winker/Degele 2010) und beeinflussen als subjektivierende Zugehörigkeitsordnungen die Lebensläufe und -wege aller Personen. Die in Herrschaftsverhältnissen immer wieder (re-)produzierten Zugehörigkeitsordnungen (vgl. Mecheril 2003) bestehen aus formalen und informellen Regelungen und Handlungspraxen. Diese Regelungen und Handlungspraxen treten systematisch auf und thematisieren, wer als ganz zugehörig, teilweise zugehörig und nicht zugehörig angesehen wird. Zugehörigkeitsordnungen bestehen u.a. in Bezug auf Geschlecht, Migrationsgesellschaft und Einkommens- sowie Behinderungsverhältnisse. Zugehörigkeitsordnungen strukturieren Lebensmöglichkeiten in Bezug auf Selbstbilder, Bewegungs-, Bildungs-, Arbeits- und Beziehungsmöglichkeiten und sind historisch und situativ wandelbar und aushandelbar (vgl. Rangger 2011).

\section{Diskriminierungs- und Rassismuskritik}

Es wird - so schlage ich in Anlehnung an Paul Mecheril vor - von diskriminierungskritisch, herrschaftskritisch oder sexismuskritisch und rassismuskritisch gesprochen und nicht von antidiskriminatorisch oder antirassistisch, um zum einen darauf hinzuweisen, dass eine komplette Abschaffung dieser Handlungspraxen und Gesellschaftsverhältnisse kaum möglich ist und zum anderen, dass es kein außerhalb von Diskriminierungs- und Herrschaftsverhältnissen gibt, sondern eine Verwobenheit auf unterschiedlichen Positionen (vgl. Mecheril 2004).

Als ein Herrschaftsverhältnis kann Rassismus definiert werden

,als ein System von Diskursen und Praxen, die historisch entwickelte und aktuelle Machtverhältnisse legitimieren und reproduzieren. Rassismus im modernen westlichen Sinn basiert auf der ,Theorie' der Unterschiedlichkeit menschlicher ,Rassen' aufgrund biologischer Merkmale. Dabei werden soziale und kulturelle Differenzen naturalisiert und somit soziale Beziehungen zwischen Menschen als unveränderliche und vererbbare verstanden (Naturalisierung). Die Menschen werden dafür in jeweils homogenen Gruppen zusammengefasst und vereinheitlicht (Homogenisierung) und den anderen als grundsätzlich verschieden und unvereinbar gegenübergestellt (Polarisierung) und damit zugleich in 
eine Rangordnung gebracht (Hierarchisierung). Beim Rassismus handelt es sich also nicht einfach um individuelle Vorurteile, sondern um die Legitimation von gesellschaftlichen Hierarchien, die auf der Diskriminierung der so konstruierten Gruppen basieren. In diesem Sinn ist Rassismus immer ein gesellschaftliches Verhältnis“ (Rommelspacher 2009).

Rassismus ist historisch untrennbar mit dem europäischen Kolonialismus verbunden, mit den zu dieser Zeit begangenen Raubzügen, Vertreibungen, Deportationen, Ermordungen sowie der wissenschaftlich für Menschen widerlegten Erfindung von „Rassen“ und deren angebliche ungleiche Wertigkeit und deren angebliches unterschiedliches Berechtigtsein auf Menschenwürde, Ressourcen und Handlungsmöglichkeiten (vgl. Plumelle-Uribe 2004). In Europa war die katholische Kirche in Verflechtung mit herrschenden Regierungen im Kontext der sogenannten Reconquista auf der iberischen Halbinsel an der Entstehung und Durchsetzung von „Rasse“-Theorien beteiligt, indem sie die Idee der „Reinheit des Blutes“ gewaltvoll durchsetzte (vgl. Fredrickson 2004). Auch im Zeitalter des Kolonialismus spielten Religionen, Missionierung mit der Ideologie der Zivilisation eine bedeutsame Rolle. Historischer und aktueller Rassismus beinhaltet gesellschaftliche Abwertungs-, Benachteiligungs- und Ausgrenzungsverhältnisse wie Rassismus gegen als Schwarze definierte Personen, antimuslimischen Rassismus (Feindlichkeit gegen Menschen als Muslime), Antiziganismus (Feindlichkeit gegen Menschen als Sinti und Roma), Antisemitismus (Feindlichkeit gegen Menschen als Jüdinnen und Juden) und einen verwertungsorientierten Nützlichkeitsrassissmus, der von der herrschenden und sich als höherwertig definierten Gruppe bestimmt wird.

\section{Wider dem herrschenden Integrationsparadigma 43}

Manuela Bojadžijev (2002) legt für das Deutschland der Nachkriegszeit anhand historischer Analysen von Widerstandsbewegungen und politischen Diskursen im Rahmen eines Streikes bei Ford in den 1970er Jahren, welcher vor allem von Arbeiter/innen mit türkischer Migrationsgeschichte getragen wurde, dar, dass das Konzept Integration von der dominanten politischen und wirtschaftlichen Klasse entwickelt wurde, um zum einen kapitalismuskritischen Widerstand durch die Trennung in „In- und Ausländer/innen“ zu spalten und zum anderen ein Täuschungskonzept gegenüber Migrant/innen zu entwickeln, welches das Versprechen von Partizipation und Teilhabe beinhaltete, jedoch nie oder nur ansatzweise umgesetzt werden sollte (Bojadžijev 
2002). Kien Ngi Ha beschreibt in postkolonialer Perspektive die historische Migrations- und Integrationspolitik der BRD seit der Nachkriegszeit als fortgesetzte, wenn auch veränderte Ausbeutung von Arbeitskraft bei gleichzeitigen Rechtseinschränkungen und Anpassungsanforderungen der als ethnisch oder rassialisierend als „Andere“ angesehenen in kolonialer Tradition (Ha 2004). Somit ist zu überlegen, ob das Konzept Integration nicht durch eine rassismuskritische Partizipations- und Teilhabepolitik zu ersetzen, zumindest ebenbürtig zu ergänzen ist.

\section{Migrantischer/Schwarzer Widerstand als Strategie und keine ausschließliche Fokussierung auf den Staat sowie seine Institutionen und seine Akteur/innen}

In der Migrationsgesellschaft gibt es unterschiedliche rassismuskritische oder antirassistische Bewegungen und Bestrebungen, die - folgen wir der Analyse von Alana Lentin (2004) - a) entweder Menschenrechte oder allgemein gleiche Rechte einfordern und primär den Staat, seine Institutionen sowie deren Akteur/innen wie Politiker/innen und Beamte adressieren oder/und b) staatliche Ausgrenzungs- und Benachteiligungspraxen auch vor dem Hintergrund der historischen Analyse von Kolonialismus und der Installierung von Nationalstaaten als zentrales Prinzip von Nationalstaaten ansehen und daher eher Personen in Veränderungsperspektive ansprechen, die von der staatlichen Gewalt und der ihrer privilegierten Bürger/innen potentiell oder tatsächlich am meisten in negativer Weise betroffen sind und am ehesten Widerstand zu leisten bereit sind. Vor allem rassistisch diskriminierte Personen können also in dieser Perspektive Veränderungen erreichen.

Da auch in historischer Perspektive Machthabende und Herrschende selten bis nie freiwillig Macht abgegeben haben, ist es - so die zweite Argumentation - mehr als fragwürdig, dass der Staat sowie seine Institutionen und seine Akteur/innen freiwillig Änderungen anstreben, die mit der Abgabe eigener Privilegien und Machtmöglichkeiten einhergeht. Vielmehr ist es historisch stets so gewesen, dass diejenigen, die am meisten negativ von Diskriminierung, Ausgrenzung, Rassismus, Sexismus oder kapitalistischer Ausbeutung betroffen sind, selbst Widerstandsformen und Taktiken (Seukwa 2007) entwickelt haben. Diese gilt es wahrzunehmen, an ihnen mitzuwirken oder sie in rassismuskritischer Perspektive - falls dies erwünscht ist - zu unterstützen. 


\section{Analyse aktueller gesellschaftlicher Rahmenbedingungen}

Gegenwärtig werden wieder einmal ethnisierende, religionsbezogene oder an „Rasse“-Konstruktionen anschließende Wir-Sie-Einteilungen in Politik und Medien eingesetzt, die möglicherweise politisch-instrumentell (Seehofer) und/oder zur Gewinnung von Autorenhonoraren vererbungsbiologische sowie rassialisierende und kulturalisierende argumentierende und mit Abwertungs- und Sanktionsvorschlägen verbundene Thesen (Sarrazin) benutzen. Immer wieder wird dazu die scheinbar neutrale und scheinbar objektive Wissenschaft herangezogen, um bestimmte Argumentationen oder Aussagen zu belegen. Vielfach erscheint in der Rede von homogen und überzeitlich konstruierten Kulturen und Kulturkreise das Konstrukt Kultur als Sprachversteck für „Rasse“-Vorstellungen (Leiprecht 2001) und zielen darauf $\mathrm{ab}$, in der Gesellschaft Gruppen zu konstruieren und voneinander zu trennen sowie gegeneinander auszuspielen. Es scheint, dass viele kritischen politischen und wissenschaftlichen Positionen und Erkenntnisse zu Mehrfachzugehörigkeiten, Hybridität, Bindestrichidentitäten aber auch zu Diskriminierungs- und Rassismusverhältnissen systematisch in die Defensive oder ins medial Off geraten. Leider sind die kultur-rassistischen oder biologisch-rassistischen öffentlich Argumentationsweisen nicht folgenlos. Sie beeinflussen Blick- und Handlungsperspektiven in der Gesellschaft und verändern oder bestärken ausgrenzende Arbeitsplatzvergabepraxen, Defizitperspektiven in der Schule und im öffentlichen Raum. Kurzum: sie re-etablieren und verfestigen rassistische Strukturen und Handlungspraxen.

Um Philomena Essed zu zitieren: Rassismus ist

„eine Ideologie, eine Struktur und ein Prozess, mittels derer bestimmte Gruppierungen auf der Grundlage tatsächlicher oder zugeschriebener biologischer oder kultureller Eigenschaften als wesensmäßig andersgeartete und minderwertige ,Rassen ' oder ethnische Gruppen angesehen werden. In der Folge dienen diese Unterschiede als Erklärung dafür, dass Mitglieder dieser Gruppierungen vom Zugang zu materiellen und nicht-materiellen Ressourcen ausgeschlossen werden“" (Essed 1992).

Wir leben in einer von Rassismus in allen Lebensbereichen beeinflussten Gesellschaft. „Racism is pervasive“ (Essed 1992), er durchdringt alle Institutions-, Lebens- und Beziehungsbereiche. Gerne wird auf öffentlichen Diskussionsveranstaltungen oder auch im Privaten von der Thematisierung von Rassismus mit dem Hinweis auf andere Themen, Ausgrenzungen und Herrschaftsverhältnisse abzulenken gesucht. Rassismus ist - und dies gilt es zu berücksichtigen - in der kapitalistischen Marktgesellschaft mit Sexismus, Behindertenfeindlichkeit, Heteronormativität verwoben. Jetzt soll es um Rassismus gehen, der untrennbar mit den anderen genannten Herrschaftsverhältnissen und Ausgrenzungspraxen verwoben ist. Die Perspektive auf Ras- 
sismus kann als Analyseperspektive gesehen werden, als ein Fenster, um mit einem Blick aus diesem Verschleierungs- und Durchsetzungstechniken des Rassismus und um Rassismus in pädagogischen Institutionen und Beratungsstellen zu erkennen.

\section{Rassismuskritische Soziale Arbeit?}

Diverse Studien zu Migration und Sozialer Arbeit (Döring/Bergmann (in: Braun 2006), Deniz (2001), Beinzger/Kallert/Kolmer (1995), Lewis (2000), Melter (2006), Kuster-Nikolić (2012)) belegen, dass Pädagog/innen wenig über die (mehrfachen) Zugehörigkeits- und Identitätsverständnisse von Jugendlichen wissen und sich wenig dafür interessieren. In Bezug auf Diskriminierungs- und Rassismuserfahrungen realisieren Pädagog/innen wenig interessierte, bis ignorante oder leugnende Handlungspraxen, die die Jugendlichen mit ihren Ausgrenzungs- und Benachteiligungserfahrungen weitgehend allein lassen. Die von den Jugendlichen der Studie „Rassismuserfahrungen in der Jugendhilfe" (Melter 2006)

„gemachten Rassismuserfahrungen waren den Pädagoginnen und Pädagogen größtenteils nicht bekannt, da sie für diese Themen kein Interesse gezeigt und in Gesprächen nicht nachgefragt haben. Wenn die Jugendlichen dennoch von Rassismuserfahrungen berichteten, wurden diese von den Pädagog/innen tendenziell geleugnet, verharmlost oder für den zu den betreuenden Jugendlichen als unproblematisch dargestellt. Die Betreuungspraxen der Pädagog/innen spiegeln die Haltung der Mehrheitsgesellschaft in Bezug auf Rassismus wider: Rassismus wird vielfach nicht thematisiert, in seiner Alläglichkeit und institutionellen Verankerung nicht wahrgenommen, individualisiert, naturalisiert, pathologisiert oder als quasi unumgängliche Folge der allgemeinen wirtschaftlichen Rezession dargestellt. Auch wird Rassismus vielfach auf physische Gewalttaten von Skinheads oder offen rassistische Beleidigungen reduziert. Gleichzeitig wird die Verantwortung für gewalttätige Konflikte nach rassistischen Beleidigungen den Opfern der verbalen Angriffe zugeschrieben.

Die fehlende Thematisierung der Rolle Sozialer Arbeit in Bezug auf institutionellen Rassismus (staatlich finanzierte Soziale Arbeit im Einsatz gegen staatliche Diskriminierung) findet bei den befragten Pädagoginnen und Pädagogen in entpolitisierten Betreuungspraxen ihren Ausdruck. Es wird deutlich, dass diese ausdrücklich betonen, sich subjekt- und lebensweltorientiert für die Interessen der Jugendlichen einzusetzen, dies im Bereich migrationssensibler und rassismuskritischer Arbeit jedoch kaum oder gar nicht praktizieren. Dies widerspricht dem gesetzlichen Auftrag des Einsatzes gegen Benachteiligung, wozu eindeutig auch Rassismus zu rechnen ist" (Melter 2009, S. 286ff.). 


\section{Ideen für eine diskriminierungs- und rassismuskritische Soziale Arbeit}

Diskriminierungs- und rassismuskritische pädagogische Handlungsempfehlungen beinhalten, so kann zusammenfassend gefolgert werden, folgende Aussagen:

- Niemand kokettiert grundlos systematisch mit eigenen Opfererfahrungen (vgl. Mecheril 2006).

- Es ist notwendig sowohl eigene Denk- und Handlungsweisen zu reflektieren als auch die eigene gesellschaftliche Position, die man/frau in Bezug auf eigene (Mehrfach-)Zugehörigkeiten hat.

- Die Funktion Sozialer Arbeit in diesen machtvollen Herrschafts- und Diskriminierungsverhältnissen ist zu reflektieren: Wie wird in „Wir“ und „Sie“ eingeteilt? Welche Gruppeneinteilungen werden durch Soziale Arbeit verstärkt oder abgeschwächt? Ist Soziale Arbeit vor allem eine Arbeit mit den $\mathrm{zu}$ „Anderen“ gemachten und benachteiligten und ausgegrenzten Personengruppen? Wie und mit welchen Zielen soll mit Adressat/innen gearbeitet werden? $\mathrm{Zu}$ wessen „Normalität“ und für welche Interessen soll erzogen und beraten werden? Gefragt werden sollte, wie strukturelle, institutionelle, diskursive und interaktive Diskriminierungs- und Herrschaftsverhältnisse im Sinne der Menschenrechtsprofession verändert werden können? - Wie können Personengruppen, die systematisch benachteiligt und ausgegrenzt werden, mehr Ausdrucks- und Handlungsmöglichkeiten bekommen? Wie kann die Teilhabe an gegebenen ausgrenzenden/benachteiligenden gesellschaftlichen Strukturen und Institutionen für diskriminierte Personengruppen ermöglicht und verbessert werden?

- Dies bedeutet die Notwendigkeit vertiefter Kenntnisse u.a. über aufenthaltsrechtliche, arbeitsmarktbezogene Gesetze sowie die Sozialgesetzgebung. Dies bedeutet die Kenntnis der historischen und aktuellen gesellschaftlichen Funktion von Diskriminierung und Herrschaft für Privilegierte und Diskriminierte auf der strukturellen, institutionellen, interaktiven, aber auch intra-psychischen Ebene.

- Dies bedeutet die Auseinandersetzung mit natio-ethno-kulturellen Mehrfachzugehörigkeiten in der Migrationsgesellschaft (Mecheril 2010) in Relation $\mathrm{zu}$ ausgrenzenden/benachteiligenden Entweder-OderDenkweisen und Gesetzen, die in der Regel von Monozugehörigkeiten ausgehen und in Deutschland vor allem deutsche Staatsbürger/innen bevorzugen. 


\section{Rassismuskritische Institutionen-analytische Selbstreflexion und Handlungspraxen}

Diskriminierungs- und Rassismuskritik kann als Beobachtungs- und Analyseperspektive auf und für Institutionen und die eigene (Team-)Arbeit dienen. In diesem Sinn soll nicht nur besprochen werden, wie es war, was wir heute oder die letzte Zeit gemacht haben. Es soll auch gefragt werden: Was machen wir hier eigentlich? Welche Aufgaben, Funktionen entsprechend welcher Zielsetzungen und mit welchen Effekten führen wir aus? Wessen Aufträgen fühlen wir uns verpflichtet und wie gehen wir mit widersprüchlichen Anforderungen in einer von Rassismus, Sexismus strukturierten kapitalistischen Leistungsgesellschaft aus? Wie arbeiten wir in einer Gesellschaft des aktivierenden Sozialstaates, der strukturelle Ungerechtigkeit mit der Ideologie der Chancengleichheit bei faktischen Diskriminierungsverhältnissen und der Reproduktion einkommensbezogener und ethnisierter Ungleichheit fortsetzt?

Migrationssensible (Teuber 2004) und rassismuskritische Soziale Arbeit strebt die Wahrnehmung der Perspektiven und Unterstützung derjenigen an, die im Alltag Rassismuserfahrungen machen und fußt auf der Kenntnis ausgrenzender Gesetzgebung und rassistischer Ausgrenzungs- und Benachteiligungspraxen. Dies kann u.a. durch die systematische Qualifizierung in Ausbildungen, die Strukturschaffung durch Jugendhilfeausschüsse, Hilfepläne und Einrichtungskonzepte und eine generelle Subjekt- und Lebenswelt- sowie Lebenslagenorientierung erfolgen, die eine Auseinandersetzung mit Alltagsrassismus (Essed 1992, Essed 2002) beinhaltet. Notwendig erscheinen systematisch organisierte und institutionell fixierte zu erstreitende Räume für die Auseinandersetzung mit institutionellen und persönlichen Konflikten und Vermeidungsstrategien gegenüber Rassismus sein. Ein Beispiel für einen ersten Raum für das Sprechen über Rassismuskritik kann durch die zeitliche, örtliche und inhaltliche Festlegung eines Settings und konkret zu besprechender oder grundsätzlicher Themen sein. Wie wäre es in Ihrer Einrichtung einen halben bis ganzen Fortbildungstag - in oder außerhalb der Einrichtung - über rassismuskritische Perspektiven und Praxen in Ihrer Einrichtung zu sprechen? Ziel kann sein, einen gleichen/ähnlichem Wissensstand zu erreichen bzw. ein Grundverständnis, ein Kernverständnis von Rassismus zu entwickeln und gemeinsame Reflexions- und Handlungsstrategien.

Angestrebt werden kann,

- dass es einen Konsens unter den Mitarbeitenden und der Leitung gibt, dass es alle sinnvoll finden, sich mit dem Thema Rassismus auseinanderzusetzen, 
- die Auseinandersetzung mit emotionalen Aspekten von Rassismuserfahrungen sowohl als Angehörige der Mehrheitsgesellschaft und auch als Personen, die Zielscheibe von Rassismen sind,

- die Sensibilisierung gegenüber Rassismuserfahrungen und das Schaffen rassismuskritischer Gesprächsräume, wo über Rassismuserfahrungen gesprochen werden kann,

- Auseinandersetzung mit aufenthaltsrechtlichen Fragen und institutionellem Rassismus und Rassismus auf dem Arbeitsmarkt und im Bildungssystem,

- Auseinandersetzung mit der eigenen Positionierung und zugeschriebenen Gruppenzugehörigkeit,

- Auseinandersetzung mit tendenziell ausgrenzenden, defizitorientierten, auf Anpassung der ethnisch Geanderten abzielenden institutionellen Aufträgen,

- Vermittlung von Wissen über die Konstruktion von Weißsein und Schwarzsein,

- die Auseinandersetzung mit Privilegien und Ausgrenzung.

In diesem Sinne sollte es in rassismuskritischer Perspektive eine Kernaufgabe werden, mit anderen Institutionen zu kooperieren und gemeinsame Förderkonzepte zu entwerfen und umzusetzen und kommunales Gesamtkonzept gegen rassistische Diskriminierung und Rassismus anzustreben.

\section{Mögliche rassismuskritische Handlungsprinzipien}

Eine rassismuskritische Perspektive kann folgende Handlungsperspektiven und grundsätzliche Prinzipien für Bildung und Erziehung beinhalten: die Reflexion und Modifikation bildungsinstitutioneller und gesamtgesellschaftlicher Prozesse und Strukturen, eine rassismuskritische Performanz mit dem Ziel, das Handeln gegen Rassismus zu stärken sowie das die Vermittlung von Wissen über Rassismus und Zugehörigkeitserfahrungen sowie die Reflexion rassistischer Zuschreibungsmuster. Ebenso beinhaltet eine rassimsukritische Perspektive die Dekonstruktion eindeutiger Unterscheidungen und die Entwicklung einer systematischen rassismuskritischen Strategie inklusive Ziele und Interventionen, die jeweils zu reflektieren sind.

„Eine konsequente rassismuskritische Perspektive wird für eine ,Lektüre' sozialer Verhältnisse eintreten, die solche Phänomene (an-)erkennt, die nicht ohne weiteres vom binären Schema erfasst und insofern von ihm ausgeschlossen werden. Praktische Anerkennung von Mehrfachzugehörigkeit und die Anerkennung von Mehrfachzugehörigkeiten sind mithin 
als bedeutsame Aspekte zu verstehen. Denn nichts widerspricht dem Rassismus so sehr wie die Mischung“" (Mecheril/Melter 2010).

\section{Literaturverzeichnis}

Badawia, Tarek (2009): Pseudodialogische Diskriminierung, in: Claus Melter/Paul Mecheril (Hg.): Rassismuskritik Band I. Rassismustheorie und -forschung, Schwalbach/Ts., S. 220-236

Beinzger, Dagmar/Kallert, Heide/Kolmer, Christine (1995): „Ich meine, man muß kämpfen können. Gerade als Ausländerin.“ Ausländische Mädchen und junge Frauen in Heimen und Wohngruppen, Frankfurt am Main

Bojadžijev, Manuela (2002): „Deutsche und ausländische Arbeiter - ein Gegner ein Kampf"? Antirassistische Kämpfe. Methodische Fragen. Historische Entwicklungen, in: Alex Demirovic/Manuela Bojadzijev (Hg.): Konjunkturen des Rassismus, Münster, S. 268-289

Bourdieu, Pierre (1992): Die verborgenen Mechanismen der Macht, Hamburg

Braun, Karl-Heinz (2006): Der Einfluss Rechtsextremer geht zurück. Forscher finden keine „national befreiten Zonen“/Rechte verbreiten dennoch Angst und Schrecken, in: Frankfurter Rundschau vom 20. Januar 2006, S. 5

Bundesministerium für Familie, Senioren, Frauen und Jugend (2009): 13. Kinder- und Jugendbericht. Bericht über die Lebenssituation junger Menschen und die Leistungen der Kinder- und Jugendhilfe in Deutschland, Berlin

Dadzie, Stella (2000): Toolkit for tackling racism in Schools, Stoke on Trent

Dirim, İnci/Mecheril, Paul (2010). Die Schlechterstellung Migrationsanderer: Schule in der Migrationsgesellschaft, in: Paul Mecheril/Maria do Mar Castro-Varela/Inci Dirim/Annita Kalpaka/Claus Melter: Migrationspädagogik, Weinheim-Basel, S. 121-138

Dittmer, Lena (2008): ,Baustein zur nicht-rassistischen Bildungsarbeit‘. Herausforderungen bei der Umsetzung in die Praxis. Untersucht am Beispiel der Bildungsarbeit in der IG Metall, Oldenburg

Deniz, Cengiz (2001): Migration, Jugendhilfe und Heimerziehung. Rekonstruktion biografischer Erzählungen männlicher türkischer Jugendlicher in Einrichtungen der öffentlichen Erziehung, Frankfurt am Main

Eckhart, Michael/Haeberlin, Urs/Sahli Lozano, Caroline/Blanc, Philippe (2011): Langzeitwirkungen der schulischen Integration. Eine empirische Studie zur Bedeutung von Integrationserfahrungen in der Schulzeit für die soziale und berufliche Situation im jungen Erwachsenenalter, Bern

ECRI (2009): ECRI-Bericht zu Deutschland, verfügbar unter: http://www.coe. int/t/dghl/monitoring/ecri/Country-by-country/Germany/DEU-CbC-IV-2009-019 -DEU.pdf (Stand: 18.12.2012)

Essed, Philomena (1992): Multikulturalismus und kultureller Rassismus in den Niederlanden, in: Rassismus und Migration in Europa, ARGUMENT-Sonderband AS 201 
Essed, Philomena (2002): Everyday Racism, in: Theo G. Goldberg/John Solomos (Hg.): A Companion to Racial and Ethnic Studies, Malden/Massachusetts Fredrickson, George M. (2004): Rassismus. Ein historischer Abriss, Hamburg Frings, Dorothee (2008): Diskriminierung wegen Religion und Weltanschauung, in: Theresia Degener/Susanne Dernne/Heike Dieball/Dorothee Frings/Dagmar Oberlies/Julia Zinsmeister (Hg.): Antidiskriminierungsrecht. Handbuch für Lehre und Beratungspraxis. Mit Lösungsbeispielen für typische Fallgestaltungen, Frankfurt am Main, S. 138-151

Frings, Dorothee/Antidiskriminierungsstelle des Bundes (2010): Diskriminierung aufgrund der islamischen Religionszugehörigkeit im Kontext Arbeitsleben - Erkenntnisse, Fragen und Handlungsempfehlungen. Diskriminierungen von Musliminnen und Muslimen im Arbeitsleben und das AGG, Berlin

Foucault, Michel (1992): Was ist Kritik?, Berlin

Gächter, August (2010): Die Verwertung der Bildung ist in allen Bundesländern das größere Problem als die Bildung selbst, verfügbar unter: https:/www.zsi.at/attach/p1509ober.pdf (Stand: 15.11.2011)

Generalversammlung der Vereinten Nationen (10. Dezember 1948): Allgemeine Erklärung der Menschenrechte. Resolution 217 A (III), verfügbar unter: http://www.un.org/depts/german/grunddok/ar217a3.html (Stand: 22.10.2012)

Gomolla, Mechthild (2005): Schulentwicklung in der Einwanderungsgesellschaft, Münster

Gomolla, Mechthild (2010): Institutionelle Diskriminierung, in: Albert Scherr/Ulrike Hormel (Hg.): Diskriminierung, Wiesbaden

Ha, Kien Nghi (2004): Ethnizität und Migration Reloaded. Kulturelle Identität, Differenz und Hybridität im postkolonialen Diskurs, Berlin

Herzog-Punzenberger, Barbara/Schnell, Philipp (2011): Bildungsforschung (in) der Migrationsgesellschaft. Entwicklungen und Perspektiven in Österreich, verfügbar unter: http://www.uni-graz.at/paedabww_schnell_herzog-punzenberger.pdf (Stand: 15.11.2011)

Hormel, Ulrike/Scherr, Albert (Hg.) (2010): Diskriminierung. Grundlagen und Forschungsergebnisse, Wiesbaden

Kessl, Fabian/Reutlinger, Christian/Ziegler, Holger (Hg.) (2007): Erziehung zur Armut? Soziale Arbeit und die ,neue Unterschicht', Wiesbaden

Kuster-Nikolić, Snežana (2012): Soziale Arbeit im Spannungsfeld des Rassismus. Erleben Migrantinnen Rassismus in der sozialarbeiterischen Beratung?, Hamburg

Leiprecht, Rudolf (2001): Alltagsrassismus. Eine Untersuchung bei Jugendlichen in Deutschland und den Niederlanden, Münster

Lentin, Alana (2004): Racism \& Anti-Racism in Europe, London

Lewis, Gail (2000): ,Race', Gender, Social Welfare. Encounters in a Postcolonial Societey, Malden

Mecheril, Paul (2003): Prekäre Verhältnisse. Über natio-ethno-kulturelle (Mehrfach-) Zugehörigkeiten, Münster-New York

Mecheril, Paul (2004): Einführung in die Migrationspädagogik, Weinheim-Basel

Mecheril, Paul (2006): Was Sie schon immer über Rassismuserfahrungen wissen wollten, in: Rudolf Leiprecht/Anne Kerber (Hg.): Schule in der Einwanderungsgesellschaft, Schwalbach/Ts. 
Mecheril, Paul (2010): Migrationspädagogik. Hinleitung zu einer Perspektive, in: Paul Mecheril/Mario do Mar Castro Varela/Inci Dirim/Anita Kalpaka/Claus Melter: Migrationspädagogik, Wiesbaden, S. 7-22

Mecheril, Paul/Melter, Claus (2010): Rassismus als machtvolle Unterscheidungspraxis, in: EEO - Enzyklopädie Erziehungswissenschaften Online. Fachgebiet Interkulturelle Bildung, herausgegeben von Hans-Joachim Roth und Charis Anastasopoulos, verfügbar unter: http://www.erzwissonline.de/\#

Melter, Claus (2006): Rassismuserfahrungen in der Jugendhilfe. Eine qualitative Studie zu Kommunikationspraxen in der Sozialen Arbeit, Münster-New York

Melter, Claus (2009): Rassismusunkritische Soziale Arbeit?, in: Claus Melter/Paul Mecheril (Hg.): Rassismuskritik Band I. Rassismustheorie und -forschung, Schwalbach/Ts.

Melter, Claus (2011a): Rezension des Buches von Jan Wulf-Schnabel (2011): Reorganisation und Subjektivierungen Sozialer Arbeit, Wiesbaden, verfügbar unter: http://www.socialnet.de/rezensionen/11152.php (Stand: 22.10.2012)

Melter, Claus (2011b): Rassismuskritische institutionelle Verantwortung als ,Luxus“ oder als Kernanliegen? Möglichkeiten, Chancen und Schwierigkeiten des Nachdenkens und Handelns von Institutionen in Bezugnahme auf Rassismus, in: Landeshauptstadt München Direktorium, Antidiskriminierungsstelle für Menschen mit Migrationshintergrund (Hg.): Dokumentation Fachtagung zu Rassismuserfahrungen, München

Melter, Claus/Karayaz, Erol (2013): Die fehlende Debatte über Diskriminierungsformen im Bildungssystem in Österreich und Deutschland, in: Paul Mecheril/Oscar Thomas-Olalde/Susanne Arens/Elisabet Romaner/Claus Melter (Hg.): Migrationsforschung als Kritik?, Wiesbaden (in Vorbereitung)

Nickel, Rainer (2010): Das Allgemeine Gleichbehandlungsgesetz in der Rechtspraxis, in: Sozialrecht + Praxis, Heft 8, S. 479-510

Plumelle-Uribe, Rosa Amelia (2004): Weiße Barbarei. Vom Kolonialrassismus zur Rassenpolitik der Nazis, Zürich

Rangger, Matthias (2011): Geänderte österreichische Jugendliche. Selbstbildarbeit in der österreichischen Migrationsgesellschaft mit dem Fokus auf (rassistische) Otheringerfahrungen, Innsbruck (unveröffentlichte Diplomarbeit)

Rommelspacher, Birgit (2009): Was ist eigentlich Rassismus?, in: Claus Melter/Paul Mecheril (Hg.): Rassismuskritik Band I. Rassismustheorie und -forschung, Schwalbach/Ts., S. 25-38

Scherr, Albert (2010): Diskriminierung und soziale Ungleichheiten. Erfordernisse und Perspektiven einer ungleichheitsanalytischen Fundierung von Diskriminierungsforschung und Antidiskriminierungsstrategien, in: Ulrike Hormel/Albert Scherr (Hg.) (2010): Diskriminierung. Grundlagen und Forschungsergebnisse, Wiesbaden, S. 36-60

Seukwa, Louis Henri (2006): Der Habitus der Überlebenskunst, Münster

Terkessidis, Mark (2004): Die Banalität des Rassismus. Migranten zweiter Generation entwickeln eine neue Perspektive, Bielefeld

Teuber, Kristin (2002): Migrationssensibles Handeln in der Jugendhilfe, in: Franz Hamburger/Gabriele Vierzigmann (Hg.): Migrantenkinder in der Jugendhilfe, München, S. 75-134 
Teuber, Kristin (2004): Migrationssensible Hilfen für Erziehung. Widersprüche aushalten und meistern, Frankfurt am Main

Winker, Gabriele/Degele, Nina (2010): Intersektionalität. Zur Analyse sozialer Ungleichheiten, Bielefeld

Wulf-Schnabel, Jan (2011): Reorganisation und Subjektivierungen von sozialer Arbeit, Wiesbaden

Zick, Andreas (1997): Vorurteile und Rassismus: eine sozialpsychologische Analyse, Münster-New York 